\title{
Enterococcus gallinarum Causing Native Valve Endocarditis
}

\author{
Teresa Dias ${ }^{1}$, José de Almeida ${ }^{2}$, Arsénio Santos ${ }^{1}$, Rui M Santos $^{1}$, Armando Carvalho $^{1}$ \\ ${ }^{1}$ Department of Internal Medicine, Centro Hospitalar e Universitário de Coimbra, Coimbra, Portugal \\ ${ }^{2}$ Department of Cardiology, Centro Hospitalar e Universitário de Coimbra, Coimbra, Portugal
}

How to cite this article: Dias T, de Almeida J, Santos A, Santos RM, Carvalho A. Enterococcus gallinarum causing native valve endocarditis. EJCRIM 2019;6: doi:10.12890/2019_001054.

Conflicts of Interests: The Authors declare that there are no competing interests.

This article is licensed under a Commons Attribution Non-Commercial 4.0 License

\section{ABSTRACT}

Endocarditis due to Enterococcus gallinarum is a rare condition, usually affecting older patients. The most frequent source of infection is the gastrointestinal or genitourinary tracts; it frequently involves the aortic valve and tends to produce heart failure.

We present a case of Enterococcus gallinarum endocarditis developing on a normal native heart valve.

Enterococcus gallinarum is intrinsically resistant to vancomycin. Antibiotic susceptibility patterns indicate that most isolates are penicillin and ampicillin-susceptible.

\section{LEARNING POINTS}

- Endocarditis due to Enterococcus gallinarum is rare.

- Enterococcus gallinarum is intrinsically resistant to vancomycin.

- Most isolates are penicillin and ampicillin-susceptible.

\section{KEYWORDS}

Endocarditis, Enterococcus gallinarum, heart failure, antibiotic

\section{INTRODUCTION}

Enterococci are well established as being a cause of endocarditis and urinary tract infections. The most common species causing human infections are Enterococcus faecalis and Enterococcus faecium; the other species are rarely encountered in human clinical specimens ${ }^{[1]}$.

Enterococcus gallinarum is part of the normal stool flora of the general population ${ }^{[2]}$.

Endocarditis due to Enterococcus gallinarum is a rare clinical condition, usually affecting older patients. The most frequent source of infection is the gastrointestinal or genitourinary tracts; it frequently involves the aortic valve and tends to produce heart failure ${ }^{[3-5]}$.

\section{CASE DESCRIPTON}

A 77-year-old woman was hospitalised with a 1-week history of progressive dyspnoea, orthopnoea, cough and lower limb oedema. The patient had prior known history of arterial hypertension.

On physical examination the patient was febrile $\left(38.5^{\circ} \mathrm{C}\right)$, polypnoeic, tachycardic and had a peripheral oxygen saturation of $88 \%$ on air, while cardiac and pulmonary auscultation revealed a $2 / 6$ pansystolic murmur loudest at the apex and bilateral pulmonary crackles. The patient also showed lower limb oedema.

The laboratory examination revealed PCR at $5.86 \mathrm{mg} / \mathrm{dl}$, without leukocytosis, $\mathrm{K}+$ at $3.1 \mathrm{mmol} / \mathrm{L}$ and BNP at $1790 \mathrm{pg} / \mathrm{ml}$. A chest X-ray showed pulmonary venous congestion and cardiomegaly. 
A transthoracic echocardiogram showed moderate to severe aortic and mitral regurgitation and vegetations with valvular destruction. The combination of vancomycin and gentamicin was started for presumably infective endocarditis.

Three days later, the patient required mechanical ventilation because of heart failure. As Enterococcus gallinarum was isolated from 2 blood cultures, gentamicin was switched to ampicillin.

Surgery was not considered because of the patient's general condition, and she died 1 month later.

\section{DISCUSSION}

Enterococcus gallinarum endocarditis is rare and, to our knowledge, this is the first report in Portugal.

Clinicians should be aware of the possibility of serious complications arising from this uncommon source of endocarditis, as early surgical management is often required for favourable outcomes ${ }^{[3]}$.

Enterococcus gallinarum is intrinsically resistant to vancomycin. Antibiotic susceptibility patterns indicate that most isolates are penicillin and ampicillin-susceptible ${ }^{[1]}$. Clinicians need to be alerted to the possibility that vancomycin may not be effective against Enterococcus gallinarum, despite in vitro results that indicate vancomycin susceptibility.

\section{REFERENCES}

1. Murray BE. Vancomycin-resistant enterococcal infections. N Engl J Med 2000;342:710-721.

2. Toye B, Shymanski J, Bobrowska M, Woods W, et al. Clinical and epidemiological significance of enterococci intrinsically resistant to vancomycin (possessing the vanC genotype). J Clin Microbiol 1997;35:3166-3170.

3. Swampillai J, Liang M, Fisher R, Devlin G. Enterococcus gallinarum causing native valve endocarditis and aorto-atrial fistula: a case report and literature review. Echocardiography 2012;29:873-875.

4. Megran DW. Enterococcal endocarditis. Clin Infect Dis 1992;15:63-71.

5. McDonald JR, Olaison L, Anderson DJ, et al. Enterococcal endocarditis: 107 cases from the international collaboration on endocarditis merged database. Am J Med 2005;118:759-766. 